\title{
SOBRE LA JUSTIFICACIÓN DE LA DEMOCRACIA REPRESENTATIVA
}

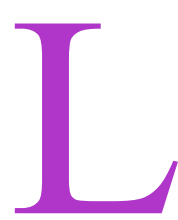

a dificultad de un breve comentario sobre los trabajos que anteceden es tan evidente que hace casi innecesaria las disculpas por la entidad (escasa) de esta nota. En efecto, las posibles sugerencias, propuestas de discusión e incluso críticas parecerían exigir, cuando menos, algo más incluso que un volumen monográfico de Doxa, pero no es ése, evidentemente, el caso. Trataré de organizar mis observaciones en torno a dos apartados: en el primero reuniré las de carácter general, en buena medida comunes a todos los artículos, y en el segundo me limitaré a enunciar casi telegráficamente algunas consideraciones particulares.

\section{I}

El orden de las aportaciones de Ruiz Miguel, Laporta, Garzón Valdés y Vidal Gil sobre democracia y representación me parece especialmente adecuado en la medida en que el trabajo del primero tiene un carácter previo, precisamente porque se sitúa (y sitúa la discusión) en el marco general, y, al mismo tiempo, por así decirlo, en el punto de salida de tal discusión en la medida en que da por supuesto que la democracia es un sistema de gobierno justo o, por decirlo en otros términos, que la democracia es un prius en lo relativo a la justificación de la dominación, al problema de la legitimidad hoy (en el sentido señalado, por ejemplo, entre nosotros, en un trabajo de hace diez años por R. Paniagua). Efectivamente, algo que parece común a los cuatro trabajos es el aceptar, desde ese punto de partida y como indica Laporta, que, a su vez, la democracia representativa no constituye una especie de sucedáneo de la «auténtica democracia», la democracia directa, que no puede calificarse este modelo como «menos democrático», sino en realidad, como algo inevitable si se quiere hablar de democracia en nuestros contextos sociales, pues se trataría de una necesidad derivada de una exigencia 
empírica y que, por consiguiente, como escribe Vidal siguiendo a Bobbio, en línea de principios no se trataría de encontrar los procedimientos para conseguir de nuevo reemplazar la democracia representativa por la directa, sino más bien de extender las leyes de la democracia representativa (pluralismo, participación y control) a la sociedad civil, lo que daría contenido a la tópica «profundización en la democracia». La verdad es que, como destacan expresamente casi todos ellos (así, por ejemplo, Garzón Valdés y Vidal) el problema, como advirtieran Montesquieu y Rousseau, es la dificultad de realizar la democracia directa fuera del contexto de sociedades homogéneas y reducidas, sociedades que, obviamente, no son las nuestras desde siglos: de ahí el paradigmático razonamiento triádico con el que Laporta resume la justificación tradicional de la representación. En el fondo, como indica Mill, a quien se recurre abundantemente, gobierno democrático sería sinónimo de «gobierno representativo». En este sentido, me ha parecido especialmente sugestivo el análisis realizado en la primera parte del trabajo de Laporta, acudiendo a una tradición sociológica y antropológica para la cual el fenómeno clave es la aparición y desarrollo de la división del trabajo. En la exposición de Laporta subyace -obiter dictumel análisis que podríamos calificar como «positivo» de la división del trabajo tal como lo introdujera Durkheim (y posteriormente, por lo que se refiere a la explicación atinente al ámbito jurídico, Kelsen, que vuelve a utilizar la metáfora biológica -órgano y función- tan frecuente en la primera etapa del desarrollo de la sociología y que, en el caso concreto de Durkheim, éste toma de Espinas, Spencer y Schaeffle). Es Durkheim, en efecto, quien comprende de modo magistral -y lo han recordado más recientemente Habermas y, entre nosotros, Pellicani en un artículo sobre el individualismo metodológico sobre el que habré de volver para comentar otras indicaciones del propio Laporta- que decir «social» es decir reglas coactivas de comportamiento, y es él quien valora el Derecho como el más importante y claro símbolo de lo social: ese es un sentido coincidente, según creo, en gran medida, con la tesis inicial de Laporta acerca de la representación, recogiendo la conocida afirmación de Honoré acerca de las normas como rasgo definitorio del grupo social (desde luego, coincidente también con la construcción de Hart), desde el rechazo explícito de Laporta del individualismo metodológico que abocaría los esfuerzos de justificación de la representación al callejón sin salida de la cláusula de imposibilidad. Sin embargo, cabría observar aquí, o mejor recordar, algo que seguramente no olvida Laporta, como tampoco, por ejemplo, 
Garzón Valdés y Vidal que expresamente lo invocan: hay otra consideración posible del fenómeno de la división social del trabajo, una consideración, desde luego, que está detrás (aun en muchos casos inconscientemente) de la crítica «de izquierda» a la representación: cuando se acepta, como lo hace Marx, que en el proceso histórico la división del trabajo es un fenómeno clave, desde luego, pero se pone en cuestión el carácter «inevitable» del sentido de la evolución de la división del trabajo, desde el análisis del proceso a través del cual ésta impondría la explotación, la alienación, proceso del que forman parte como frutos mayores de dicho fenómeno originario la aparición del Derecho y del Estado. En otras palabras, sería la división del trabajo la que impondría un modelo de organización social que, como ha recordado Capella (entre otros lugares, en las páginas de esta revista), haría aparecer como nuevo medio necesario de producción un saber científico-técnico (lo que nos recuerda Habermas con otros acentos pero no tan lejanas consecuencias para el análisis de nuestras sociedades, desde su tesis de la ciencia y la técnica como ideología) que, por su parte, da lugar a la existencia de una elite de poder -lo que será luego la clase dominante-, esto es, al conflicto nuevo en clave de clase, un conflicto que comienza en la contraposición entre asignación del trabajo y distribución del producto, de donde, finalmente, la «necesidad» de institucionalización cotidiana de la violencia que constituirían la aparición de Derecho y poder político. Por supuesto, no se trata aquí de la ingenua demanda de revisión de una y otra tradiciones, aunque quizás sí pudiera tener algún mínimo interés considerar si dentro de esa «necesidad social de diferenciación de roles» que «se da por supuesta», y que está en la base de la ineludible aparición de la representación si es que se pretende un modelo democrático, no cabría tal vez plantear la posibilidad de otros sentidos, orientaciones y manifestaciones de la evolución de la división del trabajo y, sobre todo, si, en efecto, dicha realidad social explicaría más bien sólo -como parece entender incluso Weber, aunque en el fondo retroceda- la primera fase de la aparición del fenómeno de la dominación, lo que es algo bien distinto. Por cierto que no se trata de una consideración que escape a las tesis de Laporta, para quien la representación es la consecuencia de una fase más, siempre insoslayable, de la evolución de la organización social, una consecuencia de la progresiva complejidad, heterogeneidad y atomización de los intereses propios de las sociedades modernas y del incremento casi exponencial de lo que Ruiz Miguel llama, con la tradición clásica, el ámbito personal de los sistemas políticos, del volumen de población, una tesis 
que también podemos encontrar en Durkheim y que, tal como es condensada en los epígrafes a) al d) del apartado III de Laporta constituye una línea de razonamiento coincidente en gran medida con la explicación de Hart sobre la aparición del Derecho como conjunto de reglas primarias y secundarias.

Sin embargo, y aun reconociendo la fuerza de la argumentación que ofrece Laporta, sobre todo por lo que respecta a la crítica de las construcciones que se apoyan en el individualismo metodológico, me parece que la explicación que ofrece Garzón Valdés (y que, en buena parte, es complementaria de la de Laporta) siguiendo las tesis de Kelsen, sería más satisfactoria e incluso más consistente con el punto de partida de ambos trabajos (también de los de Ruiz Miguel y, en otra medida, del de Vidal): la valoración del principio de autonomía individual como supuesto en el que se asienta el mismo fundamento de la democracia: lo que Laporta llama el individualismo ético, el principio de no dictadura de Arrow invocado a su vez por Garzón tras la huella de Mill al establecer el criterio básico de la libre elección de planes de vida. Efectivamente, el parlamentarismo sería un compromiso entre la exigencia democrática de libertad y la división del trabajo impuesta por las modernas sociedades nacionales, y la representación no sería más que la ficción que trata de legitimarlo desde el punto de vista de la soberanía popular (Kelsen la califica, en otro lugar, como ideología -en el sentido preciso de construcción no científica- cuya función es «ocultar la situación real y mantener la ilusión de que el legislador es el pueblo, a pesar de que en realidad la función del pueblo -o, dicho más correctamente, del cuerpo electoral- se encuentra limitada a la creación del órgano legislativo»). El eje del compromiso, como indica Garzón, sería la clave: el principio de mayoría (distinto del domino de la mayoría) más la protección de las minorías, lo que exigiría conceptualmente el catálogo de derechos humanos. Pero sobre esto habrá que volver después.

Creo que otra dificultad -que no me arriesgo a considerar objeción- en los planteamientos de Laporta y sobre todo de Garzón Valdés (me parece que también de Vidal) es precisamente la que deriva de admitir que el supuesto fáctico de la inevitabilidad de la representación es la existencia de sociedades heterogéneas, y, al mismo tiempo, en el plano normativo (no es ésta la única contradicción entre ambos planos: por ejemplo, aunque también sobre ello volveremos, entre el marco analítico y el normativo en lo que respecta a las tesis de la democracia económica y a las exigencias éticas no utilitaristas) se proponen unos criterios que exigirían un modelo de sociedades dotadas de 
un mínimo -quizá no tan pequeño, si se lee bien- grado de homogeneidad social: en Garzón Valdés esto es consecuencia de adoptar las tesis de Kelsen (aunque también es un punto de vista que se encuentra en Burke y Schmitt, pese a las diferencias en los planteamientos, derivadas sobre todo del relativismo axiológico de Kelsen frente al objetivismo platónico de aquéllos): en definitiva, la heterogeneidad, sobre todo la heterogeneidad económico-social, es una amenaza para la posibilidad de la «condición de esperanza» y el requisito de equilibrio de poder que Garzón Valdés extrae de Buchanan y Heller, a pesar de que parece haber una restricción de dicho grado de homogeneidad a lo que Garzón Valdés llama el «coto vedado». No tengo tan claro que la homogeneidad económico-social (la redistribución de la riqueza, la igualdad material, la equiparación en el punto de partida) esté incluida de la misma forma entre las condiciones que, según Laporta, legitimarían como justa a una institución social, si bien hay que reconocer que incluye en el punto 3.e) una fórmula que parece atender a ello, aunque la generalidad del enunciado parece insuficiente: «tratar a todos por igual» (que es tanto -y tan poco- como decir «dar a cada uno lo suyo»). Algo similar sucede con las tesis de Ruiz Miguel, sobre todo cuando afirma que «los derechos a la igualdad y libertad en esferas no políticas como ... la igualdad laboral» pueden «ser violados sin riesgo para la limpieza del propio juego democrático», y también con las de Vidal, aunque en él, cuando sigue las directrices de Bobbio, parece observarse alguna precaución adicional (por ejemplo, en base a la distinción de Bobbio entre pequeño y gran mercado, o al invocar las críticas de Marx), pero, si se me permite decirlo en otros términos, tampoco en ese trabajo aparece una consideración acerca de las contradicciones entre el mercado (o, más genéricamente, el capitalismo) como modo de producción y la asamblea de representantes, la democracia representativa como forma legítima de dominación. Ya Mill, sin embargo, se debatía en el convencimiento de tal incompatibilidad, como puede verse con claridad en su propuesta de sistema de voto plural (circunstancia que, por cierto, cuando se invoca a Mill en los trabajos que comento, solamente menciona Ruiz Miguel) al paso de su conocida reflexión sobre el gobierno representativo: me refiero al voto de calidad de empresarios, banqueros y comerciantes para compensar el desequilibrio del voto de la mayoría desprovista de recursos (desde luego, sí que se afirma expresamente en todos los artículos -jfaltaría!- el requisito del voto igual universal, pero no se recuerda la posición de Mill). Hoy, desde luego, parecería que el capitalismo fuera el único sistema compatible 
con la democracia (polacos y húngaros entregados como conversos a pregonarlo, para satisfacción de no pocos: recuérdense las afirmaciones de Walesa) desde una perspectiva que parece reafirmar aún más la virtualidad de la aplicación del análisis económico en el ámbito político. Ejemplos de esto último son la utilización por Laporta de la concepción económica de la democracia de Schumpeter (no sólo en el artículo que comento: ya ha desarrollado brillantemente la aplicación de ese instrumental teórico en otros trabajos) y Downs en el marco analítico, y la de Buchanan y también en cierto modo de Gauthier por parte de Garzón Valdés. A este respecto, habría que admitir que hoy la concepción de Schumpeter (la democracia como elección global entre diversas opciones en competencia por el voto) es mucho más útil desde el punto de vista de la comprensión de la realidad que las fórmulas de Lincoln, pero no es menos cierto que ese approach metodológico (como seguramente le gustaría decir a Garzón Valdés) comporta no pocos riesgos, sobre todo cuando se trata de formular propuestas, cuando se pasa al plano normativo: de un lado, esa visión económica del elector como cliente puede abocar, como en las últimas propuestas de Brenan (un colaborador crítico, pero estrecho, de Buchanan) a un análisis de las expectativas y criterios de decisión que pueden desproveer de fundamento racional a la democracia misma, al disolver el mecanismo básico de la representación, el voto -su motivación- en mero cálculo de beneficio individual en el que los intereses generales, la solidaridad, la igualdad o la distribución de la riqueza brillan por su ausencia, cuando no, ante la imposibilidad de prever eficazmente tal beneficio, en la adhesión característica de las manifestaciones de masa, en la mera expresión de un sentimiento de simpatía (desde luego en el sentido fuerte del término, lo que daría para otro comentario) similar al de un supporter en el fútbol británico. De otra parte, como subrayan sin embargo los cuatro y muy claramente Laporta, aparece el riesgo de sustituir el debate democrático por un juego de negociación entre corporaciones, en el que los intereses de los ciudadanos pueden ser sustituidos -o simplemente inducidos artificialmente- por los de los grandes agentes económicos.

Otra de las posibles observaciones, que afectaría a la justificación misma de la representación, es la relativa a la relación entre representantes y representados. Aquí planean entre otras dificultades, como advierte Ruiz Miguel, la propia determinación del concepto de «afectados» (sobre lo que volveré en II), pero, sobre todo, la ambigüedad de la noción misma de representación. Aunque, en este sentido, el análisis de Laporta (con el que coincide 
no poco Vidal, siguiendo a Bobbio) partiendo de la taxonomía de Pitkin es, en mi opinión, básico, subsisten por mi parte algunas dudas que afectarían a la justificación misma de la representación. Si aceptamos como descripción de la realidad la inversión del mecanismo de representación propio del Derecho privado (en la tipología de Laporta, la representación 3 y la 4) realizada por el Derecho público -porque, como advierte Bourdeau, «el papel de los representantes no es expresar una voluntad que sería preexistente en el cuerpo nacional. Es, según los términos que resurgen sin cesar en las obras de los técnicos franceses de la democracia, querer por la nación»- se plantea el problema de qué se representa: Laporta rechaza la respuesta que remite a los intereses de los representados, porque entiende que lo importante son las decisiones sobre los intereses y su gestión, y no la determinación del concreto interés de cada uno, y por ello desplaza el problema a las cuestiones de la representación como autorización y del control periódico de los representantes, para concluir la naturaleza esencialmente formal o procedimental de la representación. Por su parte, Garzón Valdés, tras subrayar el punto de vista de Burke sobre la incompetencia básica de las propuestas de los representados («opiniones»), estima que la clave es, por el contrario, la noción de intereses, si bien corregida: la «idea correcta» de interés que pudiera permitir no un consentimiento fáctico - un compromiso- sino hipotético, en línea con Habermas, hasta llegar a incluir la noción de deseos secundarios, compatibles con la existencia de un coto vedado y que serían susceptibles de la misma justificación ética que los intereses particulares en Habermas. Entiendo que la noción de interés no es eliminable del concepto de representación, y que no hay tanta diferencia real entre las posiciones de Laporta y Garzón Valdés. En efecto, con Laporta, habría que destacar el problema de los intereses generales, lo que acerca su posición a las otras, pero además, si bien, con Ruiz Miguel, es necesario reconocer que la justificación de la democracia depende del contenido de la justificación y no de la forma o método utilizado, y, con Garzón Valdés, que no basta con una justificación instrumental de la representación, no creo que la propuesta de Laporta sea meramente instrumental: él mismo relativiza esa afirmación desde el momento en que invoca condiciones procedimentales básicas que son «de inequívoca naturaleza racional o moral», y por lo que respecta al procedimiento de designación de representantes exige rasgos como «la igual consideración de los afectados, la libertad de acción y decisión, un amplio intercambio de opiniones y el principio mayoritario», a lo que habría que añadir el sentido 
no meramente formal de la periodicidad en la rendición/control de cuentas por parte de los representados (y recordamos el paso de Aristóteles sobre el significado e importancia de la alternancia en el poder).

Por otra parte, en la relación entre representantes y representados subsisten dos problemas a los que los autores que preceden prestan desigual atención: el primero de ellos es el de la progresiva burocratización de los representantes (y la consiguiente ley de bronce de Michels) y el segundo el de la apatía/indiferencia y ausencia de control por parte de los representados. Ambos tendrían un origen común: la creciente dificultad y por ello la especialización en la gestión de los asuntos públicos. En el fondo, es el problema ya denunciado en el famoso pasaje de Rousseau sobre el sistema parlamentario inglés (que cita Vidal). Es verdad que, por ejemplo, como señala también Vidal, el problema de la creciente complejidad de la decisión política sitúa en un grado de incompetencia (y proporcionalmente de distanciamiento) a los representados (basta pensar en el recurso a técnicos y burócratas en las comisiones del Parlamento), por no hablar de la responsabilidad de los aparatos de partido en las decisiones tanto primarias como de lo que Ruiz Miguel llama, siguiendo a Dahl, «control de la agenda», las metadecisiones: cabría recordar que si bien no hay mandato imperativo, sí existen ab initio listas cerradas e, in exertitio, disciplina de voto. Por lo demás, parece subyacer un planteamiento cercano al paternalismo en algunas tomas de posición de Ruiz Miguel y Garzón Valdés (riesgo denunciado, por cierto, por Laporta: «en el ámbito de la política no parece que tenga justificación alguna el definir la representación sin prever un lugar destacado en la misma para las preferencias expresadas de los representados»). Por ejemplo, cuando el primero exige respecto a las metadecisiones «consciencia de su posibilidad y oportunidad», o cuando plantea el problema de las metametadecisiones. Por su parte, Garzón Valdés subraya en exceso, a mi modo de ver, la incompetencia básica de los representados, paradójicamente en línea con Burke (al que critica con su habitual perspicacia) en base al carácter subjetivo y voluble de las «opiniones» de los representados, en contraposición con sus intereses que, sin embargo, en la construcción de Garzón Valdés nunca son realmente, según me parece, tales intereses propios de los representados: los primarios, porque en realidad son objetivos e independientes de su voluntad (los bienes básicos); los secundarios, porque no deben ser formulados expresamente, sino supuestos racionalmente por los representantes desde un consentimiento hipotético de los representados 
«a lo Habermas» si se me permite la expresión, aunque el propio Garzón Valdés advierte al respecto contra un «paternalismo legislativo injustificable». Sin embargo, debo añadir inmediatamente que la formulación que ofrece Ernesto Garzón Valdés sobre el núcleo de la actividad del representante (me refiero a la tesis inicial del apartado III.2.f: «el ámbito de gestión del representante es, por una parte el del afianzamiento de la vigencia efectiva de los bienes básicos... pero también el campo de la negociación y el compromiso») me parece casi enteramente suscribible con sólo algunas matizaciones que recogeré a continuación.

Por último, en lo que concierne (y dentro de las limitaciones propias: no pretendo que éstas sean las únicas consideraciones posibles, desde luego) a estas observaciones generales, restaría el problema de los límites al ámbito de decisión que diría Ruiz Miguel, o, en expresión de Garzón Valdés, el problema del «coto vedado». Si aceptamos el presupuesto al que me he referido con anterioridad, está claro que existiría un grupo de cuestiones que afectan a la institución de la representación y quedan fuera del alcance de disposición de los representantes. Sería así, por ejemplo, en el caso de las metametadecisiones, aunque con algún matiz que trataré de precisar en el apartado II. Sería así, desde luego, en lo tocante a lo que Garzón Valdés llama el coto vedado, y ello porque (Ruiz Miguel, invocando a Elías Días) la democracia no agota la legitimidad ni la justicia: los bienes básicos para la realización del plan de vida, en línea con Rawls (bienes primarios) o intereses universalizables, por seguir a Habermas. Estos se caracterizan, además, por una condición expansiva, respecto a los cuales sería indiferente el consenso fáctico de representados o representantes, y cuya vigencia efectiva es independiente de la voluntad, deseos o intereses de los integrantes de la comunidad, porque no aceptarlos (y esto es un razonamiento que me parece a su vez paternalista) sería prueba de irracionalidad, de incompetencia. Lógicamente, respecto a los elementos integrantes del coto vedado, subsisten buena parte de los interrogantes relativos al concepto y fundamentación de los derechos humanos (que, por supuesto, Garzón Valdés no pretende resolver aquí): en primer lugar su propio catálogo, pues ¿cuáles son los derechos que se han de incluir en él? ¿Cómo se establecen o mejor, cómo se encuentran y conocen? A todo esto hay que añadir la contradicción entre el carácter pretendidamente universal e imparcial de esos derechos y la situación real, de donde resulta necesario concluir que, o bien no es cierto que no haya sociedades justas sin su reconocimiento y establecimiento, o 
acordar que este paradigma de sociedad justa pertenece al orden de los sueños que, como recuerda Ruiz Miguel, es algo gratuito. Sucede aquí, en mi opinión, lo mismo que con las condiciones expresadas por Laporta, que resultarían aceptables en tanto que horizonte normativo, pero difícilmente permitirían aceptar como justa ninguna institución social (y, sobre todo, la democracia representativa) existente en la actualidad. En relación con este problema y con el anterior, me parece especialmente relevante algo que ya propuse en su momento a Laporta (como se ve, sin demasiado éxito): la introducción del principio de publicidad como condición de justificación de la democracia representativa (especialmente de la representativa). En efecto, tanto Garzón Valdés como Vidal recogen expresamente la crítica de Schmitt sobre ese punto en relación con el funcionamiento del pleno del Parlamento y, posteriormente, cuando se hace eco Garzón Valdés de la concepción del Parlamento en Kelsen como prosecución de la línea media entre mayoría y minorías, como garantía para oír la voz de las minorías y para que haya posibilidad de control de la mayoría, sobre todo cuando ésta es absoluta (en ese caso el único control posible es precisamente el de la publicidad y el recurso a los instrumentos privilegiados de la misma significativamente la prensa y la radio y televisión). En ese sentido, como he tratado de argumentar en otros lugares, la publicidad aparecería como una de las condiciones o elementos lógicos de la democracia, exigencia incrementada para hacer posible el control de la gestión de los representantes, el verdadero debate parlamentario, la garantía de los derechos y de la existencia del pluralismo. Como barrera, pues, de un lado, contra la tendencia a la burocracia y la especialización que devienen casi forzosamente en el secreto, $\mathrm{y}$, de otra parte, como antídoto frente al problema de la abstención que señala Ruiz Miguel, la apatía y el desinterés de los ciudadanos por las cosas públicas, el peligro que ya entrevieran Mill y sobre todo (como señala R. Paniagua) Tocqueville, una tendencia que acaba venciendo la propia soberanía política democrática, porque ésta sólo permite salir un momento de la dependencia e indiferencia (como Rousseau: el momento de acudir a las urnas), mientras que aquella «los conduce a renunciar al uso de su voluntad; extingue así poco a poco su espíritu y enerva su alma».

\section{II}

Se me permitirá, para terminar, tan sólo algunas brevísimas 
consideraciones más particulares en relación con cada uno de los trabajos:

1. En el ensayo de Ruiz Miguel tendría algunas dudas sobre las siguientes cuestiones:

a) Es verdad que en el intento de delimitar la noción clave de «afectados» se desecha el criterio subjetivo en aras del objetivo, pero permanece el problema de la definición. ¿Cómo establecer quién es el afectado? Recuerdo dos contraejemplos formulados por Garzón Valdés, el caso del ciudadano panameño respecto a las elecciones presidenciales en Estados Unidos (al que alude Ruiz Miguel) y el del millonario respecto a la huelga de RENFE. Tampoco el criterio de pertenencia nacional (criterio de «membrecía») lo resuelve, como advierte también Ruiz Miguel, que indica él mismo los contraejemplos de los extranjeros en las elecciones locales y el más dudoso aun de las «generaciones futuras».

b) En la medida en que se sostiene que, sobre todo cara a los conflictos que plantea el ámbito local (o autonómico) de la democracia «termina apareciendo la vieja fórmula del Estado nacional» como instancia ulterior decisoria, no acabo de comprender las razones que le hacen optar, en el ámbito mundial, por una organización internacional centralizadora cuyas decisiones, para ser democráticas «deberían seguir el camino de la igualdad individual y no estatal».

c) Finalmente, me parece que las nociones de compromiso y pacto de un lado, y unanimidad o consenso de otro, tal como las formula Ruiz Miguel, inducen a algunos problemas conceptuales que se resolverían si se adoptase el uso de esos términos que, en el mismo contexto, propone Garzón Valdés, tras haber examinado, por ejemplo, las diferencias entre las posiciones de Schmitt, Kelsen y Habermas al respecto.

2. En lo que hace al artículo de Laporta, me limitaré a dos cuestiones que me parecen de especial interés:

a) En lo que toca a la tipología de la representación, me parece que habría que señalar en primer lugar que, en realidad, la dimensión jurídica de la representación no se limita a la representación-3. En realidad, bien pensado (y lo digo porque creo que el propio Laporta así lo sostenía expresamente, al menos inicialmente), las especies 3, 4 y 5 de la representación no son más que tres momentos de la misma, relativos al origen, contenido y resultado de la misma, de forma que podría presentarse la representación como un subsistema normativo (con normas de origen, cambio y organización). Por lo demás, no estoy tan seguro de que sea tan sencillo descartar la representación-reflejo 
(la representación-2): en primer lugar, porque el criterio material no es tan absurdo (y basta pensar en el régimen de designación de presidente en las comunidades de vecinos, o en formas de composición de asambleas por mero sorteo o turno); en todos esos supuestos, si se conserva la alternancia y la rendición de cuentas no se ve por qué razón este sistema ha de ser necesaria mente más propicio a la «representación virtual», a la suplantación de la voluntad de los representantes (¿y qué significa la tesis de Michels?), lo que, por otra parte, matizaría también la objeción de imposibilidad de puesta en práctica de los métodos demoscópicos.

b) Me parece asimismo fundamental la revisión de las tesis del individualismo metodológico y, sobre todo, del deslizamiento inconsciente desde el individualismo ético al metodológico. Entiendo que el intento de evitar el problema de la justificación a través de la cláusula de imposibilidad es correcto, porque el problema de fondo es la dificultad de resolver desde esos paradigmas metodológicos los problemas de la lógica de la acción colectiva, pero no acabo de ver cómo se concilia el instrumental metodológico y conceptual (adecuado para el momento descriptivo) propio del análisis económico, con las ineludibles exigencias normativas, sobre todo si se pretende superar la ética utilitarista (a recordar lo obvio: que el individualismo metodológico, con la hipótesis básica del egoísmo racional y la maximalización de beneficios se contrapone expresamente a la perspectiva normativa). Por lo demás, no estoy muy seguro de la vía media que escoge Laporta, acudiendo a las interacciones regladas de los individuos para evitar tanto las explicaciones puramente subjetivistas como la hipostatización de lo colectivo. Creo que la dirección ofrecida por Durkheim, suficientemente corregido el sacrificio de lo individual, o, si se quiere así, que la propuesta de la perspectiva relativista-subjetivista- teleológica que ofrece Ferrari respecto a la explicación funcionalista podrían ofrecer otras perspectivas quizás más adecuadas.

3. Es cierto que a lo largo de este comentario he suscrito en gran medida las tesis de Ernesto Garzón Valdés, lo que explica, por otra parte, la telegráfica brevedad de las observaciones siguientes:

a) Cuando Garzón Valdés sostiene el carácter expansivo de los bienes básicos (y no sé si con ello trata de reproducir desde el punto de vista ontológico la tesis clásica del Bonum diffusivum sui, y, desde el punto de vista cognoscitivo, la tesis de Suárez de la posibilidad de cambio por incremento del contenido del Derecho natural), me gustaría conocer su posición respecto al dilema 
planteado por Laporta respecto a la ampliación del catálogo de derechos (por ejemplo, los derechos de la tercera y sucesivas generaciones), y la correlativa pérdida de fuerza de los mismos.

b) En relación con la «lectura» de Kelsen que nos presenta Garzón, subsisten dudas acerca del sentido en el que puede hablarse en Kelsen de «derechos fundamentales». Parece incuestionable cuál sea la posición de Kelsen respecto a la necesidad de respetar los derechos de la minoría en relación con el juego democrático (y significativamente el derecho a hacerse oír) para que haya respeto al principio de mayoría y no simplemente dominio de la mayoría, pero eso parece estar un tanto lejos de la afirmación de los derechos fundamentales individuales como coto vedado a la discusión y por ende a la producción normativa.

4. Finalmente, destacaré un problema en la argumentación de mi compañero el profesor Vidal: creo que el planteamiento de la cuestión de la obligación política (de la obediencia al Derecho, tal como él lo formula) no está tan inseparablemente vinculado como él lo plantea al problema de la representación. Creo que la respuesta que ofrece Laporta a las críticas formuladas en ese sentido (hay referencia expresa a la posición de Pateman) es clara: la cuestión del fundamento de vinculatoriedad aparecería como un problema independiente, o, al menos, si se refiere así, para resolver la cuestión relativa a quién debe representar no es necesario plantearse la segunda. 